\title{
Multi-frequency Spin Manipulation Using Rapidly Tunable Superconducting Coplanar Waveguide Microresonators
}

\author{
A. T. Asfaw, ${ }^{1,}$ a) A. J. Sigillito, ${ }^{1}$ A. M. Tyryshkin, ${ }^{1}$ T. Schenkel, ${ }^{2}$ and S. A. Lyon ${ }^{1}$ \\ ${ }^{1)}$ Department of Electrical Engineering, Princeton University, Princeton, New Jersey 08544 , \\ $U S A$ \\ ${ }^{2)}$ Accelerator Technology and Applied Physics Division, Lawrence Berkeley National Laboratory, Berkeley, \\ California 94720, USA
}

(Dated: 27 September 2018)

In this work, we demonstrate the use of frequency-tunable superconducting NbTiN coplanar waveguide microresonators for multi-frequency pulsed electron spin resonance (ESR) experiments. By applying a bias current to the center pin, the resonance frequency $(\sim 7.6 \mathrm{GHz})$ can be continuously tuned by as much as 95 $\mathrm{MHz}$ in $270 \mathrm{~ns}$ without a change in the quality factor of 3000 at $2 \mathrm{~K}$. We demonstrate the ESR performance of our resonators by measuring donor spin ensembles in silicon and show that adiabatic pulses can be used to overcome magnetic field inhomogeneities and microwave power limitations due to the applied bias current. We take advantage of the rapid tunability of these resonators to manipulate both phosphorus and arsenic spins in a single pulse sequence, demonstrating pulsed double electron-electron resonance (DEER). Our NbTiN resonator design is useful for multi-frequency pulsed ESR and should also have applications in experiments where spin ensembles are used as quantum memories.

Superconducting microresonators have dramatically enhanced the detection sensitivity of conventional electron spin resonance (ESR). Recently, single-shot detection of $10^{7}$ spins has been demonstrated at $2 \mathrm{~K}$ using coplanar waveguide $(\mathrm{CPW})$ resonators $\frac{112}{12}$, and further improvements in the readout at $\mathrm{mK}$ temperatures have enabled detection of $10^{3}$ spins ${ }^{3 / 4}$ using lumped-element resonators. As a result, these resonators are routinely used for the manipulation of spin ensembles and have also been proposed as buses for coherently coupling superconducting qubits with spins $\frac{566}{}$. The resonance frequencies of $\mathrm{CPW}$ and lumped-element resonators are typically fixed by fabrication, and conventional methods used to tune the resonance frequency post-fabrication using superconducting quantum interference devices are incompatible with the high magnetic fields that are typically necessary for X-band ESR. A method that enables frequency tunability of superconducting microresonators at high magnetic fields is thus desirable for multifrequency ESR. The ability to tune the resonance frequency on-demand also allows greater control of spin dynamics ${ }^{7 / 8}$ as well as the coupled spin-cavity dynamics in the strong-coupling regime where spin ensembles are used as quantum memories ${ }^{619}$. In this work, we fabricate frequency-tunable CPW resonators that are compatible with high magnetic fields following recently developed techniques ${ }^{10} 12$. We show multi-frequency ESR with ${ }^{31} \mathrm{P}$ donors in ${ }^{28} \mathrm{Si}$, finding no effect on spin coherence when the resonators are operated at different frequencies. We show that the resonance frequency can be tuned in no more than $\sim 270 \mathrm{~ns}$. As a practical application, we take advantage of the rapid tunability of our resonators to manipulate ${ }^{31} \mathrm{P}$ and ${ }^{75} \mathrm{As}$ donor spin ensembles that are $33 \mathrm{MHz}$ apart in a single pulse sequence.

a) Electronic mail: asfaw@princeton.edu
The resonators are fabricated from a $20 \mathrm{~nm}$ NbTiN thin film $\left(T_{C}=13.8 \mathrm{~K}\right)$ on a c-axis sapphire substrate using optical lithography followed by reactive-ion etching with an $\mathrm{SF}_{6} / \mathrm{Ar}$ plasma. Devices are wirebonded to a copper printed circuit board equipped with microwave connectors and attached to a rotatable sample holder which enables in situ alignment of the device with its surface parallel to the externally applied magnetic field.

An optical micrograph of a device is shown in Fig. 17. The structure is a photonic bandgap (PBG) resonator with two Bragg mirrors on either side of a cavity which defines the resonance frequency. A key feature of the PBG resonator is that the center pin is continuous throughout the device, enabling the application of bias currents. Further details of the PBG resonator design have been discussed previously 2113 . The Bragg mirrors are constructed using four periods of stepped-impedance waveguides, where a period is defined as a length of lowimpedance $(35 \Omega)$ followed by a length of high-impedance $(137 \Omega)$ transmission line. The top inset of Fig. 1 1 shows a magnified view near one of the impedance steps. Three devices are fabricated where the width of the center pin of the cavity is $1.5,2.5$ and $4 \mu \mathrm{m}$. Care is taken to prevent current crowding $\frac{14 \mid 15}{15}$ at both ends of the cavity by tapering from the impedance steps, as shown in the bottom inset of Fig. 1a.

Microwave transmission through each device is monitored at a temperature of $2 \mathrm{~K}$ while a bias current is applied to the center pin. With no bias current, the resonance frequency of the $4 \mu \mathrm{m}$ device is $7636.6 \mathrm{MHz}$ (Fig. 1b) with a temperature-limited loaded quality factor of $\sim 3000$ and a coupling coefficient of 0.6 . With $5 \mathrm{~mA}$ of current applied to this device, the resonance frequency shifts to $7584.3 \mathrm{MHz}$, indicating a frequency shift, $\delta \mathrm{f}=$ $52 \mathrm{MHz}$. The device maintains its quality factor as the bias current is increased from $0 \mathrm{~mA}$ to $5 \mathrm{~mA}$. The critical current of the device is measured to be $5.014 \mathrm{~mA}$, 
in agreement with previously reported values for similar NbTiN wires where current crowding was minimized 14 .

Fig. 17 shows the dependence of $\delta \mathrm{f}$ as a function of the applied bias current for all three devices. The $1.5 \mu \mathrm{m}$ device shows the largest change in resonance frequency, $\delta \mathrm{f}=95 \mathrm{MHz}$, while the $2.5 \mu \mathrm{m}$ device shows a change of $\delta \mathrm{f}=78 \mathrm{MHz}$ before the applied current exceeds the critical current of the superconductor.

The resonance frequency shift follows a well-known dependence on the applied bias current $\frac{1016}{}$. The total inductance of our transmission lines is composed of both geometric and kinetic inductance. The application of a bias current, $i$, modulates the kinetic inductance, resulting in a change from the zero-current resonance frequency, $\mathrm{f}_{0}$, according to the expression

$$
\frac{\delta \mathrm{f}(i)}{\mathrm{f}_{0}}=-\left[\left(\frac{i}{I_{2}^{*}}\right)^{2}+\left(\frac{i}{I_{4}^{*}}\right)^{4}\right]
$$

where we note that the response is always negative, independent of the sign of the current $\frac{16}{16}$. Here, $I_{2}^{*}$ and $I_{4}^{*}$ are parameters that set the scale of the current nonlinearity of the kinetic inductance. We fit the measured values of $\delta \mathrm{f}$ for our three devices and extract $I_{2}^{*}=\{28.9,50.1,62.5\} \mathrm{mA}$ and $I_{4}^{*}=\{13.9,26.3,35.7\}$ $\mathrm{mA}$ for the $\{1.5,2.5,4\} \mu \mathrm{m}$ devices, respectively. The $1.5 \mu \mathrm{m}$ device shows the largest response to applied current since it has the largest kinetic inductance fraction of the three devices. While the quartic component has typically been small in previous reports $12[17$, we find that it is necessary when biasing near the critical current. Such dependence has been reported in devices with large kinetic inductance fractions 1118 .

Next, we evaluate the ESR performance of our devices using a sample consisting of a $2 \mu \mathrm{m}$ epitaxial layer of isotopically enriched ${ }^{28} \mathrm{Si}$ grown on high-resistivity p-type $\mathrm{Si}$. The epi-layer is bulk-doped with ${ }^{31} \mathrm{P}$ donors to a concentration of $5 \times 10^{15} / \mathrm{cm}^{3}$ and implanted with ${ }^{75} \mathrm{As}$ donors at a nominal depth of $200 \mathrm{~nm}$ to a concentration of $5 \times 10^{16}$ donors $/ \mathrm{cm}^{3}$ as discussed in Ref. 2, We clip the sample on top of the $4 \mu \mathrm{m}$ resonator with a phosphor bronze spring. We choose this resonator over the $1.5 \mu \mathrm{m}$ and $2.5 \mu \mathrm{m}$ devices because of better expected homogeneity of the microwave magnetic field seen by donor spins in the $2 \mu \mathrm{m}$ epi-layer.

The device and sample are cooled to $2 \mathrm{~K}$ in a magnetic field of $B_{0}=275 \mathrm{mT}$ oriented parallel to the plane of the superconductor. This orientation maintains the loaded quality factor of 3000 by avoiding dissipation due to the motion of trapped flux vortices in the superconductor under the influence of microwave currents 1920. We measure echo-detected field sweeps of ${ }^{31} \mathrm{P}$ donors with and without bias currents as shown in Fig. 2. In these measurements, the intensity of a Hahn echo $(\pi / 2(+\mathrm{x})-\tau-\pi(+\mathrm{y})-\tau-$ echo $)$ is monitored with $\tau=60 \mu \mathrm{s}$ as the external magnetic field, $B_{0}$, is swept. Fig. 2a shows the results of field sweeps measured without bias current, where the $m_{I}=-1 / 2{ }^{31} \mathrm{P}$ donor hy- (a)

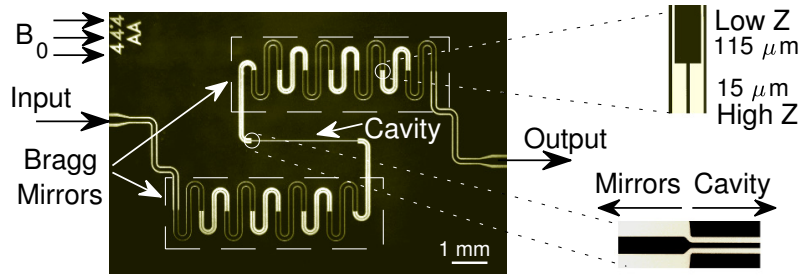

(b)
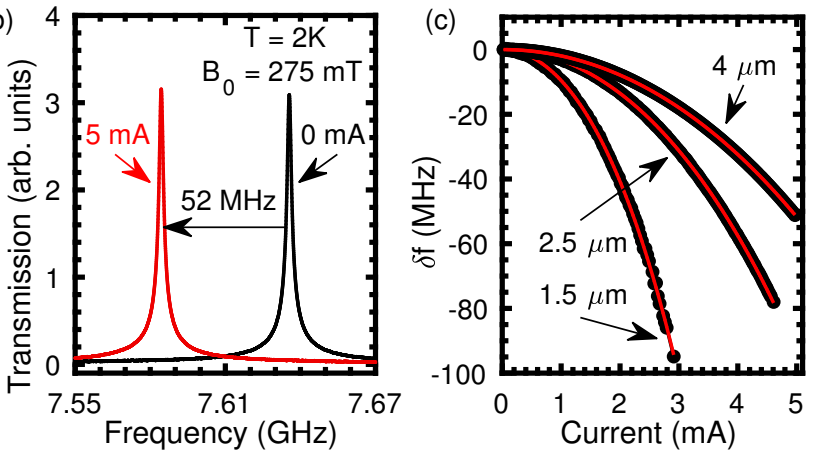

FIG. 1. (a) Optical micrograph of a tunable superconducting coplanar photonic bandgap microresonator. The device is patterned from a $20 \mathrm{~nm} \mathrm{NbTiN} \mathrm{thin} \mathrm{film} \mathrm{on} \mathrm{a} \mathrm{c-axis} \mathrm{sapphire}$ substrate. The photonic bandgap is defined by the two Bragg mirrors on either side of the cavity. The Bragg mirrors are implemented using stepped impedances (top inset) and the resonance frequency is determined by the length of the cavity. The stepped impedances are tapered at both ends of the cavity to avoid current crowding (bottom inset). The center pin is continuous throughout the device from the input port to the output port. Three such devices are investigated with cavity center pin widths of $1.5 \mu \mathrm{m}, 2.5 \mu \mathrm{m}$ and $4 \mu \mathrm{m}$. (b) The resonance frequency of the $4 \mu \mathrm{m}$ device shifts by $\delta \mathrm{f}=52$ $\mathrm{MHz}$ for a bias current of $5 \mathrm{~mA}$, with no change in the quality factor of 3000 . (c) Dependence of $\delta \mathrm{f}$ on bias current for the three different devices. The maximum value of $\delta \mathrm{f}$ before the bias current exceeds the critical current is seen to be 95 $\mathrm{MHz}, 78 \mathrm{MHz}$ and $52 \mathrm{MHz}$ for the $1.5 \mu \mathrm{m}, 2.5 \mu \mathrm{m}$ and $4 \mu \mathrm{m}$ devices, respectively. The fits (red lines) are generated using Eq. 1 .

perfine line appears at $274.78 \mathrm{mT}$. We repeated this experiment using both rectangular $(\pi / 2$-pulse $=200 \mathrm{~ns}, \pi$ pulse $=400 \mathrm{~ns}$ ) and adiabatic pulses (BIR4-WURST20 1 , $10 \mu \mathrm{s}$, chirp frequency $\pm 2 \mathrm{MHz}$ ) with microwave powers of $-29 \mathrm{dBm}$ and $-32 \mathrm{dBm}$, respectively, at the input port of the resonator. Adiabatic pulses have previously been shown to compensate for rotation errors arising from microwave magnetic field inhomogeneities in the bulk-doped sample ${ }^{1}$. As shown in Fig. 2a, the signals obtained using rectangular and adiabatic pulses are comparable, indicating good homogeneity of the microwave magnetic field in the $2 \mu \mathrm{m}$ epi-layer.

We performed the field sweep experiment with a bias current of $i=4 \mathrm{~mA}$ applied to the center pin of the resonator throughout the entire pulse sequence. The ${ }^{31} \mathrm{P}$ signal is shifted to $273.72 \mathrm{mT}$ in agreement with the resonance frequency change of $33 \mathrm{MHz}$. The center pin of the device is aligned with the external magnetic field, $B_{0}$, such that the inhomogeneous magnetic field gener- 
ated by the applied bias current, $B_{i}$, mostly adds to $B_{0}$ in quadrature. However, a small misalignment $\left(B_{0}\right.$ in the plane of $\mathrm{NbTiN}$ but misaligned with the long axis of the resonator) leads to a component of $B_{i}$ along $B_{0}$ that broadens the observed ESR transition for spins directly above the center pin. Such a misalignment does not affect spins above the gap between the center pin and the ground plane where $B_{i}$ remains orthogonal to $B_{0}$ with the misalignment. From simulations that take into account the full distribution of $B_{i}$ and the microwave magnetic field, $B_{1}$, we estimate that a broadening comparable to the ESR linewidth can be caused by a misalignment of $\sim 4.7^{\circ}$. Additionally, we observed that microwave powers larger than $-32 \mathrm{dBm}$ affected the resonance lineshape with a bias current of $4 \mathrm{~mA}$ due to the non-linearity of the superconductor ${ }^{21 \mid 22}$. The reduced signal intensity for rectangular pulses is due to a combination of reduced microwave power and broadening due to misalignment. By using adiabatic pulses which are not power-limited at -32 $\mathrm{dBm}$, we nearly recover the full signal intensity as shown in Fig. 2 b.

In order to evaluate the effect of the applied bias currents on the coherence of the spin ensemble, we measure $T_{2}$ with and without bias currents as shown in Fig. 2 . In both cases, we use adiabatic pulses and measure the intensity of the Hahn echo as the interpulse delay, $\tau$, is increased. We find $T_{2}=448 \pm 5.2 \mu \mathrm{s}$ without bias current and $T_{2}=450 \pm 9.1 \mu$ s with $4.9 \mathrm{~mA}$ bias current. The agreement of these two values indicates that there is no effect on spin coherence due to noise in the applied bias current at timescales shorter than $\sim 1 \mathrm{~ms}$. It is conceivable that the stability of the current source will become important at longer timescales.

In Fig. 3, we measure how quickly we can tune the resonator to a new frequency by applying a current pulse to the center pin and monitoring microwave transmission through the device. As the resonance frequency shifts in response to the current pulse at $t=0$, it coincides with the frequency of the probe tone at a later time, $t=T$, enabling transmission through the resonator and resulting in a peak in the measurement at time $T$. We show the results of this measurement for a $3.9 \mathrm{~mA}$ current pulse in which the resonator reaches the target $\delta \mathrm{f}=31.2 \mathrm{MHz}$ in $270 \mathrm{~ns}$. The inset compiles all measured tuning times for the $4 \mu \mathrm{m}$ and $1.5 \mu \mathrm{m}$ devices showing no frequency dependence with an average delay of 270 ns. In our experiments, we used resistive power combiners and DC blocks to combine the microwaves with the bias currents. The measured tuning times are likely limited by the time constant of our current-biasing circuit rather than the response of the superconductor.

We demonstrate the use of this rapid tunability in a double electron-electron resonance (DEER) experiment $23 \mid 24$. Here, we monitor the Hahn echo intensity from ${ }^{31} \mathrm{P}$ donors while also applying a $\pi$ pulse to ${ }^{75} \mathrm{As}$ donors (hereafter referred to as $\pi_{\mathrm{As}}$ ). The pulse sequence is shown in the inset of Fig. 4. The effect of $\pi_{\mathrm{As}}$ is to flip ${ }^{75}$ As donors such that the local dipolar field which is seen
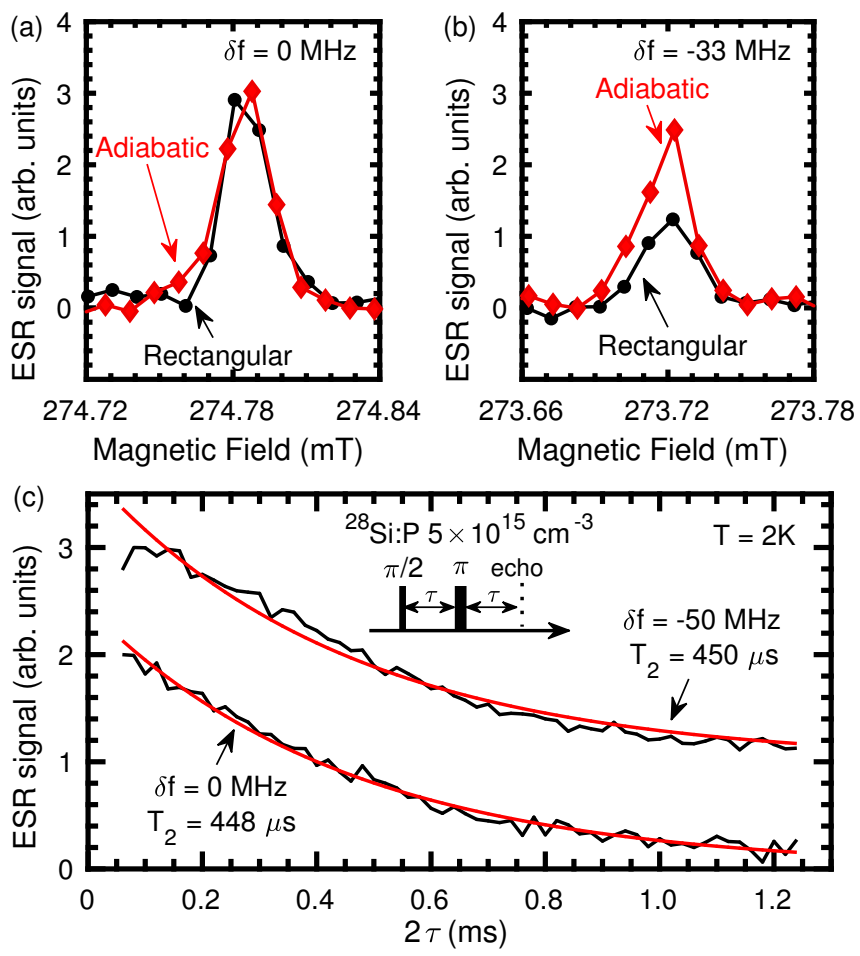

FIG. 2. Echo-detected field sweeps of the $m_{I}=-1 / 2 \mathrm{ESR}$ transition of ${ }^{31} \mathrm{P}$ donor spins in ${ }^{28} \mathrm{Si}$ with $0 \mathrm{~mA}$ (a) or $4 \mathrm{~mA}$ (b) of bias current applied to the center pin of the resonator. Rectangular and adiabatic pulses are tested in both cases, and are found to produce similar signal intensities when no current flows through the center pin. On the other hand, rectangular pulses show reduced signal intensity when current flows through the center pin. The nonlinearity of the superconductor limits the maximum microwave power that can be applied at the input port of the resonator in the presence of a bias current. The reduced echo intensity for rectangular pulses is due to a combination of the reduced microwave power and magnetic field inhomogeneities introduced by the applied bias current. Adiabatic pulses are not power-limited and nearly recover the full signal intensity. (c) Coherence times are found to be the same with and without bias current, indicating that the bias current noise does not introduce additional decoherence to the spins at timescales shorter than $\sim 1 \mathrm{~ms}$.

by each ${ }^{31} \mathrm{P}$ donor spin in the first free evolution period $(\tau=34 \mu \mathrm{s})$ is different from that of the second. This results in additional dipole-induced dephasing of the ${ }^{31} \mathrm{P}$ donor spin ensemble due to the random distribution of ${ }^{75}$ As donors; the effect is maximized when $\pi_{\mathrm{As}}$ is applied close to the ${ }^{31} \mathrm{P}$ donor $\pi$-pulse ${ }^{25}$.

The $\pi_{\text {As }}$ pulse is applied at time $t$ after the $\pi / 2$ pulse which tips the ${ }^{31} \mathrm{P}$ donors. The minimum value of $t$ is $6 \mu \mathrm{s}$, arising from timing delays that protect the detection circuitry of our spectrometer and a $1 \mu \mathrm{s}$ wait time for the resonator to settle to the frequency of ${ }^{75} \mathrm{As}$ donors before the application of $\pi_{\mathrm{As}}$. The results are shown in Fig. 4. where $\pi_{\mathrm{As}}$ is either on or off resonance with ${ }^{75} \mathrm{As}$ donors, keeping the rest of the experimental parameters constant. When $\pi_{\mathrm{As}}$ is resonant, a reduction in the Hahn 


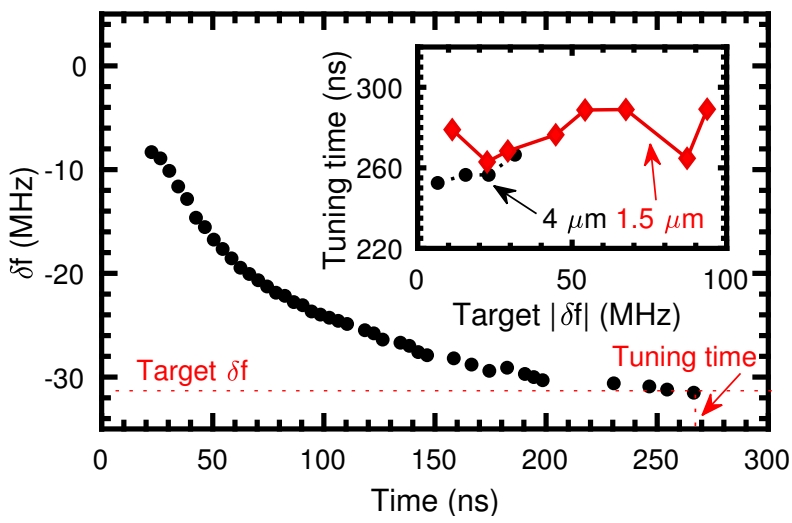

FIG. 3. Dynamics of the resonance frequency change in response to a $3.9 \mathrm{~mA}$ current pulse at time $t=0$ measured by monitoring microwave transmission through the device. The inset shows tuning times for various values of the target $\delta \mathrm{f}$ for the $4 \mu \mathrm{m}$ and $1.5 \mu \mathrm{m}$ devices. The measured tuning times are independent of the frequency change.

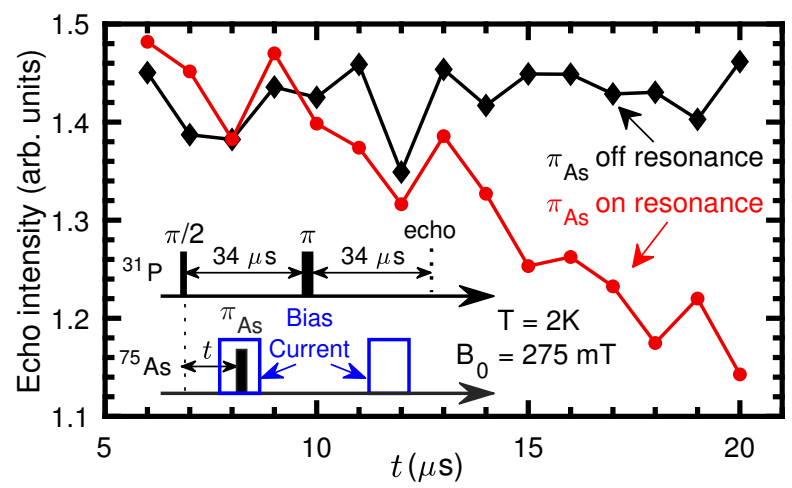

FIG. 4. Double electron-electron resonance with ${ }^{31} \mathrm{P}$ and ${ }^{75} \mathrm{As}$ spin ensembles. The Hahn echo from ${ }^{31} \mathrm{P}$ spins is monitored while a $\pi$-pulse (labeled $\pi_{\mathrm{As}}$ ) is applied to the ${ }^{75} \mathrm{As}$ spins. The position $t$ of this pulse is swept and its effect on the ${ }^{31} \mathrm{P}$ spin echo is measured. The two spin transitions are $33 \mathrm{MHz}$ apart. Details about the pulse sequence are described in the main text. When $\pi_{\text {As }}$ is resonant with the ${ }^{75}$ As spins, dipoleinduced dephasing of the ${ }^{31} \mathrm{P}$ spins is enhanced and the echo intensity is reduced by $\sim 20 \%$.

echo intensity of $\sim 20 \%$ at $t=20 \mu \mathrm{s}$ is observed due to additional dephasing in the ${ }^{31} \mathrm{P}$ donors arising from changes in the local magnetic field environment when the ${ }^{75}$ As donor spins are flipped.

We note here that a typical fixed-frequency resonator used for DEER requires a low Q-factor of $\sim 230$ in order to accommodate the two donor frequencies that are 33 $\mathrm{MHz}$ apart. This is disadvantageous because the use of a low-Q resonator requires high-power pulses to invert both donors, and also leads to poor detection sensitivity. In contrast, our tunable resonator maintains a Q-factor of 3000 at both donor frequencies. The use of a fixedfrequency resonator would lead to a reduction in the detected echo signal by a factor of $\sim 10$ meaning an increase in averaging time by a factor of $\sim 100$ to maintain a similar signal-to-noise ratio. Therefore, our tunable resonator enables high-sensitivity DEER with significantly reduced signal-averaging time.

We remark here on practical considerations when using these devices. (1) The large kinetic inductance nonlinearity of NbTiN results in an undesirable limitation on the maximum microwave power that can be applied for spin manipulation. For our $4 \mu \mathrm{m}$ and $2.5 \mu \mathrm{m}$ devices, microwave powers at the input port of the resonator beyond -15 and $-23 \mathrm{dBm}\left(B_{1}=0.2\right.$ and $\left.0.09 \mu \mathrm{T}\right)$, respectively, were seen to affect the resonance lineshape without bias currents. This behavior has been reported previously ${ }^{2122}$, and is a signature of the strong nonlinearity of the superconductor. (2) In our DEER experiments, we applied two bias current pulses - one before, and another after the $\pi$ pulse on ${ }^{31} \mathrm{P}$ donors - to cancel phase accumulation from the inhomogeneous magnetic fields generated by the bias currents. We have found that bipolar current pulses can also be used to compensate for the unwanted phases since the negative currents reverse phase accumulation due to the positive currents. Bipolar pulses are advantageous because they only need to be applied on one side of the $\pi$ pulse. (3) Pulsed DEER experiments for biochemical samples frequently must flip broad lines 26 . Recent experiments have used chirped adiabatic pulses 27 , but very low-Q resonators are then required, resulting in poor sensitivity. The tunable high-Q resonator frequency could be varied during the pulse to follow the chirped pulse frequency, thus allowing broad lines to be inverted without compromising the sensitivity of the experiment.

Our frequency-tunable devices may also be useful in experiments where a spin ensemble that is strongly coupled to the resonator is used as a quantum memory ${ }^{6}$. This enables storage of quantum information for timescales of order $T_{2}^{*}$ of the spin ensemble ${ }^{28}$ and can, in principle, be extended to $T_{2}$, which can exceed second $\sqrt{29}$ in silicon, by the use of refocusing $\pi$ pulses. However, the application of a $\pi$ pulse also inverts the spin ensemble into an unstable state that can emit the stored photons into the cavity by superradiance $9128 \mid 30$. In order to avoid uncontrolled emission of photons into the cavity, the resonator can be detuned away from the ESR frequency after the $\pi$ pulse ${ }^{30}$. In this work, we have demonstrated the desired frequency tunability. Further work is needed to elucidate the effect of magnetic field inhomogeneities introduced by the bias currents on the coupled spin-cavity dynamics.

In summary, we have demonstrated frequency-tunable ESR microresonators fabricated from NbTiN by taking advantage of the non-linear kinetic inductance of the superconductor. We have shown the ability to rapidly tune the resonance frequency of these devices by as much as $\delta \mathrm{f}=95 \mathrm{MHz}$ within $270 \mathrm{~ns}$ while maintaining a quality factor of 3000 at $7.6 \mathrm{GHz}$ in a magnetic field of $275 \mathrm{mT}$ at $2 \mathrm{~K}$. We evaluated the performance of these resonators for ESR, finding that adiabatic (BIR4-WURST20) pulses 
can be used to overcome magnetic field inhomogeneities and microwave power limitations due to the applied bias current. We find that the applied bias currents have no effect on spin coherence at timescales up to $1 \mathrm{~ms}$. We take advantage of the rapid frequency tunability to demonstrate high-sensitivity double electron-electron resonance in which we address both ${ }^{31} \mathrm{P}$ and ${ }^{75} \mathrm{As}$ donor spin transitions that are separated by $33 \mathrm{MHz}$ in a single pulse sequence. Our devices enable multi-frequency electron spin resonance with CPW microresonators and may also be used in a hybrid quantum computation platform where spin ensembles that are strongly coupled to our resonator serve as quantum memories.

We thank Dr. Robin Cantor for preparation of NbTiN thin films. Devices were fabricated in the Princeton Institute for the Science and Technology of Materials Micro/Nano Fabrication Laboratory and the Princeton University Quantum Device Nanofabrication Laboratory. Work at Princeton was supported by the NSF through the Princeton MRSEC (Grant No. DMR-01420541) and by the ARO (Grant No. W911NF-13-1-0179). Work at LBNL was performed under the auspices of the U.S. Department of Energy under Contract No. DE-AC02$05 \mathrm{CH} 11231$.

${ }^{1}$ A. J. Sigillito, H. Malissa, A. M. Tyryshkin, H. Riemann, N. V. Abrosimov, P. Becker, H.-J. Pohl, M. L. W. Thewalt, K. M. Itoh, J. J. L. Morton, A. A. Houck, D. I. Schuster, and S. A. Lyon, Appl. Phys. Lett. 104, 222407 (2014)

${ }^{2}$ A. J. Sigillito, A. M. Tyryshkin, T. Schenkel, A. A. Houck, and S. A. Lyon, arXiv:1701.06650 [quant-ph] (2017)

${ }^{3}$ C. Eichler, A. J. Sigillito, S. A. Lyon, and J. R. Petta, Phys. Rev. Lett. 118, 037701 (2017)

${ }^{4}$ A. Bienfait, J. J. Pla, Y. Kubo, M. Stern, X. Zhou, C. C. Lo, C. D. Weis, T. Schenkel, M. L. W. Thewalt, D. Vion, D. Esteve, B. Julsgaard, K. Mølmer, J. J. L. Morton, and P. Bertet, Nat. Nanotechnol. 11, 253 (2016)

${ }^{5}$ K. D. Petersson, L. W. McFaul, M. D. Schroer, M. Jung, J. M. Taylor, A. A. Houck, and J. R. Petta, Nature 490, 380 (2012)

${ }^{6}$ Y. Kubo, C. Grezes, A. Dewes, T. Umeda, J. Isoya, H. Sumiya, N. Morishita, H. Abe, S. Onoda, T. Ohshima, V. Jacques, A. Dréau, J.-F. Roch, I. Diniz, A. Auffeves, D. Vion, D. Esteve, and P. Bertet, Phys. Rev. Lett. 107, 220501 (2011).

${ }^{7}$ H. Kaupp, T. Hümmer, M. Mader, B. Schlederer, J. Benedikter, P. Haeusser, H.-C. Chang, H. Fedder, T. W. Hänsch, and D. Hunger, Phys. Rev. Applied 6, 054010 (2016)
${ }^{8}$ A. Bienfait, J. J. Pla, Y. Kubo, X. Zhou, M. Stern, C. C. Lo, C. D. Weis, T. Schenkel, D. Vion, D. Esteve, J. J. L. Morton, and P. Bertet, Nature 531, 74 (2016)

${ }^{9}$ C. Grezes, B. Julsgaard, Y. Kubo, M. Stern, T. Umeda, J. Isoya, H. Sumiya, H. Abe, S. Onoda, T. Ohshima, V. Jacques, J. Esteve, D. Vion, D. Esteve, K. Mølmer, and P. Bertet, Phys. Rev. X 4, 021049 (2014)

${ }^{10}$ A. J. Annunziata, D. F. Santavicca, L. Frunzio, G. Catelani, M. J. Rooks, A. Frydman, and D. E. Prober, Nanotechnology 21, $445202(2010)$

${ }^{11}$ M. R. Vissers, J. Hubmayr, M. Sandberg, S. Chaudhuri, C. Bockstiegel, and J. Gao, Appl. Phys. Lett. 107, 062601 (2015)

${ }^{12}$ A. A. Adamyan, S. E. Kubatkin, and A. V. Danilov, Appl. Phys. Lett. 108, 172601 (2016)

${ }^{15}$ Y. Liu and A. A. Houck, Nature Phys. 13, 48 (2017)

${ }^{14}$ H. L. Hortensius, E. F. C. Driessen, T. M. Klapwijk, K. K. Berggren, and J. R. Clem, Appl. Phys. Lett. 100, 182602 (2012) http://dx.doi.org/10.1063/1.4711217

${ }^{15}$ J. R. Clem and K. K. Berggren, Phys. Rev. B 84, 174510 (2011)

${ }^{16}$ J. Zmuidzinas, Annu. Rev. Condens. Matter Phys. 3, 169 (2012)

${ }^{17}$ J. Luomahaara, V. Vesterinen, L. Grönberg, and J. Hassel, Nat. Commun. 5, 4872 (2014)

${ }^{18}$ A. Kher, P. K. Day, B. H. Eom, J. Zmuidzinas, and H. G. Leduc, J. Low Temp. Phys. 184, 480 (2016)

${ }^{19}$ V. Ginzburg and L. Landau, Zh. Eksperiment. Teoret. Fiz. 20, 1064 (1950)

${ }^{20}$ H. Malissa, D. I. Schuster, A. M. Tyryshkin, A. A. Houck, and S. A. Lyon, Rev. Sci. Instrum. 84, 025116 (2013)

${ }^{21}$ B. Abdo, E. Segev, O. Shtempluck, and E. Buks, J. Phys.: Conf. Ser. 43, 1346 (2006)

${ }^{22}$ L. J. Swenson, P. K. Day, B. H. Eom, H. G. Leduc, N. Llombart, C. M. McKenney, O. Noroozian, and J. Zmuidzinas, J. Appl. Phys. 113, 104501 (2013)

${ }^{23}$ A. Milov, K. Salikhov, and M. Shirov, Fiz. Tverd. Tela 23, 975 (1981)

${ }^{24}$ A. Milov, A. Ponomarev, and Y. D. Tsvetkov, Chem. Phys. Lett. 110, 67 (1984).

${ }^{25}$ A. D. Milov, A. G. Maryasov, and Y. D. Tsvetkov, Appl. Magn. Reson. 15, 107 (1998)

${ }^{26}$ O. Schiemann and T. F. Prisner, Q Rev Biophys. 40, 1 (2007)

${ }^{27}$ I. Niemeyer, J. H. Shim, J. Zhang, D. Suter, T. Taniguchi, T. Teraji, H. Abe, S. Onoda, T. Yamamoto, T. Ohshima, J Isoya, and F. Jelezko, New J. Phys. 15, 033027 (2013).

${ }^{28}$ B. C. Rose, A. M. Tyryshkin, H. Riemann, N. V. Abrosimov, P.Becker, H.-J. Pohl, M. L. W. Thewalt, K. M. Itoh, and S. A. Lyon, arXiv:1702.00504 [quant-ph] (2017)

${ }^{29}$ A. M. Tyryshkin, S. Tojo, J. J. L. Morton, H. Riemann, N. V. Abrosimov, P. Becker, H.-J. Pohl, T. Schenkel, M. L. W. Thewalt, K. M. Itoh, and S. A. Lyon, Nat. Mater. 11, 143 (2012)

${ }^{30}$ B. Julsgaard and K. Mølmer, Phys. Rev. A 88, 062324 (2013) 